Pacific Journal of Mathematics

ON THE CRITICAL LINES OF A GRAPH

and Michael David Plumier
and 


\title{
ON THE CRITICAL LINES OF A GRAPH
}

\author{
Lowell W. Beineke, Frank Harary,
} and Michael D. Plummer

A set of points $M$ is said to cover a graph $G$ if every line in $G$ has at least one point in $M$. Call $M$ a minimum cover (m.c.) for $G$ if it is a point cover with a minimum number of points. The number of points in any minimum cover of a graph $G$ is called the point covering number of $G$ and is denoted by $\alpha(G)$. If $x$ is a line in $G$, denote by $G-x$ the graph obtained from $G$ by deleting $x$. Similarly, if $v$ is a point of $G, G-v$ will denote the graph obtained from $G$ by deleting $v$ and all lines incident with $v$, A line $x$ in $G$ is said to be a critical line (with respect to point covering) if $\alpha(G-x)<$ $\alpha(G)$. A graph is called line-critical if every line is critical. Obviously every complete graph is line-critical, and so is any odd cycle. There are, however, many other line-critical graphs.

The main purpose of this paper is to prove that in any graph, two adjacent critical lines must lie on an odd cycle. This result will imply that a line-critical graph must be a block and furthermore, that any two adjacent lines in such a graph must lie on an odd cycle.

This condition, though necessary for a graph to be line-critical, is not sufficient as is illustrated by the graph in Figure 1.

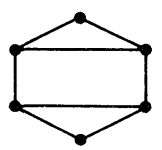

FIGURE 1 .

The concept of a line-critical graph is introduced by Ore [4] and briefly considered by Erdös and Gallai [3]. However, a structural characterization of line-critical graphs remains unknown, although one of the authors [5] constructs an infinite family of such graphs which, in particular, includes all line-critical graphs with fewer than eight points.

2. Additional terminology. A graph $G$ consists of a finite nonempty set of points $V(G)$ and a set of lines $E(G)$ each of which is an unordered pair of points. The line $u v$ is incident with each of its points. Two points (lines) joined by a line (point) are said to be adjacent. Let $|A|$ denote the number of elements in the set $A$. The degree of a point $v$ is the number of lines incident with it and is denoted by $d(v)$. A line is called an endline if one of its points has 
degree one. A point $v$ is isolated if $d(v)=0$.

A walk $W$ of $G$ is an alternating sequence of points and lines, beginning and ending with points (said to be joined by $W$ ) such that each line is incident with the points before and after it. A walk $P$ is a path if its points are distinct. The length of a walk is the number of occurrences of lines in it. If $W$ is a walk beginning and ending with the same point, $W$ is said to be closed. A closed walk in which all points are distinct is called a cycle.

The graph $G$ is connected if every two points are joined by a path. A point $v$ is a cutpoint of the connected graph $G$ if $G-v$ is disconnected. If $v$ is any point of a graph $G$, a branch of $G$ at $v$ is a maximal connected subgraph of $G$ not having $v$ as a cutpoint. A connected graph is a block if it has no cutpoints. If $G$ is a graph and $H$ is a subgraph of $G$, then $G-H$ is the subgraph of $G$ defined by (1) $V(G-H)=V(G)$ and (2) $E(G-H)=E(G)-E(H)$.

3. Preliminary results. The main result of this paper will be obtained in the following manner. First it will be shown that any two critical lines which are adjacent must lie on a cycle. Next in Theorem 2 it is proved that if the graph is bipartite, then no two critical lines are adjacent. Then based on the assumption that every cycle containing two adjacent critical lines is even, a contradiction to Theorem 2 is obtained. Finally, it is pointed out that since the point covering number is additive on the components of a graph, it may be assumed without loss of generality that all graphs are connected.

THEOREM 1. Any two adjacent critical lines of a graph lie on a cycle.

Proof. Suppose the conclusion is false, so that there are adjacent critical lines $x$ and $y$ in $G$ which do not lie on a common cycle. Let $x=u w$ and $y=v w$. Then $w$ is a cutpoint of $G$. Partition the lines of $G$ as follows. Let $H$ be the branch of $G$ at $w$ which contains $x$ and let $J$ be the branch containing $y$. Hence $G$ has the general appearance of Figure 2.

Let $M$ be an m.c. for $G-x-y$. Then $M \cup\{w\}$ covers $G$. Thus $\alpha(G-x-y)=\alpha(G)-1$, and hence $\alpha(G-x)=\alpha(G-x-y)=\alpha(G-y)$.

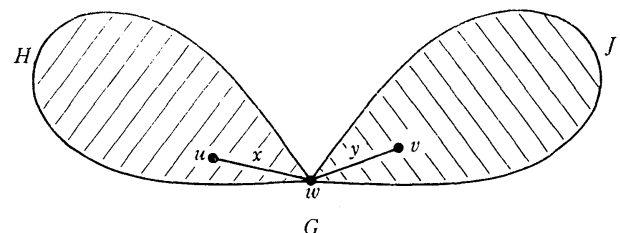

FIgURe 2. 
Thus every m.c. for $G-x$ and every m.c. for $G-y$ is also an m.c. for $G-x-y$. Let $M_{x}$ be an m.c. for $G-x$. Hence $u, w \notin M_{x}$ and $v \in M_{x}$. Similarly if $M_{y}$ is an m.c. for $G-y$, then $u \in M_{y}$, and $v, w \notin M_{y}$.

Next it is shown that $M_{x} \cap V(J)$ is an m.c. for $J$ by assuming the contrary. Then there is a set $N \subset V(J)$ which covers $J$ but with $|N|<\left|M_{x} \cap V(J)\right|$. Hence $W=N \cup\left[M_{x} \cap V(H)\right] \cup\{w\}$ covers $G$ and it follows that

$$
\begin{aligned}
\alpha(G) & \leqq|W|=\left|N \cup\left[M_{x} \cap V(H)\right] \cup\{w\}\right|=|N \cup\{w\}|+\left|M_{x} \cap V(H)\right| \\
& \leqq|N|+1+\left|M_{x} \cap V(H)\right| \leqq\left|M_{x} \cap V(J)\right|+\left|M_{x} \cap V(H)\right| \\
& =\left|M_{x}\right|=\alpha(G-x),
\end{aligned}
$$

thus contradicting the hypothesis that $x$ is critical in $G$. Similarly, $M_{y} \cap V(H)$ is an m.c. for $H$.

Next it will be shown that $R=\left[M_{x} \cap V(J)\right] \cup\left[M_{y} \cap V(H)\right]$ is an m.c. for $G-x-y$. Clearly $R$ covers $G-x-y$. Let $S$ be any m.c. for $G-x-y$. Now if $w \in S$, then $S$ covers $G$ and hence $\alpha(G) \leqq$ $\alpha(G-x-y)$ which is a contradiction. Hence $w \notin S$ and thus $S \cap V(H)$ and $S \cap V(J)$ are disjoint sets. Now it is shown that $S \cap V(H)$ is an m.c. for $H$ by assuming the contrary. Then there is a set $U$ which covers $H$ with $|U|<|S \cap V(H)|$. Now $\mathrm{w} \in U$ or else $S$ is not an m.c. for $G-x-y$. Hence $U \cup[S \cap V(J)]$ covers $G$. Thus

$$
\begin{aligned}
\alpha(G) & \leqq|U|+|S \cap V(J)|<|S \cap V(H)|+|S \cap V(J)| \\
& =|S|=\alpha(G-x-y)
\end{aligned}
$$

which is absurd. Thus $S \cap V(H)$ is an m.c. for $H$. Similarly, $S \cap V(J)$ is an m.c. for $J$. Hence

$$
\begin{aligned}
|S| & =|S \cap V(H)|+|S \cap V(J)|=\alpha(H)+\alpha(J) \\
& =\left|M_{x} \cap V(J)\right|+\left|M_{y} \cap V(H)\right|=|R|
\end{aligned}
$$

and hence $R$ is an m.c. for $G-x-y$. But $u \in M_{y} \cap V(H)$ and $v \in M_{x} \cap V(J)$, hence $u, v \in R$. Thus $R$ covers $G$ and $\alpha(G) \leqq|R|=$ $\alpha(G-x-y)$ again contradicting the hypothesis that $x$ is critical in $G$. This completes the proof of the theorem.

The next theorem is a corollary to the results obtained by Dulmage and Mendelsohn $[1,2]$. However, since a direct proof is short, it is included for the sake of completeness.

THEOREM 2. No two critical lines of a bipartite graph are adjacent.

Proof. Let $G$ be a bipartite graph and suppose $G$ contains two adjacent critical lines $x=u w$ and $y=w v$. Let the two point sets of 
of $G$ be $S$ and $T$, and let $M_{x}$ and $M_{y}$ be m.c.'s for $G-x$ and $G-y$ respectively. Let $S_{x}=S \cap M_{x}, S_{y}=S \cap M_{y}, T_{x}=T \cap M_{x}$, and $T_{y}=$ $T \cap M_{y}$. It may be assumed without loss of generality that $u, v \in S$ and $w \in T$. Now $u, w \notin M_{x}$, but $v \in M_{x}$, while $v, w \notin M_{y}$, but $u \in M_{y}$.

Since $M_{x} \cup\{w\}$ and $M_{y} \cup\{w\}$ both cover $G, \alpha(G-x)=\alpha(G-y)=$ $\alpha(G-x-y)$. Thus $M_{x}$ and $M_{y}$ are both m.c.'s for $G-x-y$. But then by Theorem 5 of [1], $N=S_{x} \cup S_{y} \cup\left(T_{x} \cap T_{y}\right)$ is also an m.c. for $G-x-y$. Thus $N$ contains both $u$ and $v$ and hence $N$ covers $G$. Therefore $\alpha(G) \leqq \alpha(G-x-y)=\alpha(G-x)$, contradicting the hypothesis that $x$ is critical in $G$, and thus completing the proof.

4. The main theorem. The principal result of this paper may now be proved.

THEOREM 3. Every two adjacent critical lines of a graph lie on an odd cycle.

Proof. Let $x=u w$ and $y=v w$ be adjacent critical lines in $G$. By Theorem 1, there is at least one cycle in $G$ containing both $x$ and $y$. Consider all cycles of $G$ containing both lines $x$ and $y$. To prove the theorem, assume that all such cycles are even.

ASSERTION 1. If $H$ is the subgraph of $G$ induced by all the lines of the cycles containing both $x$ and $y$, other than $x$ and $y$ themselves, then $H$ is bipartite.

To prove this assertion, let $C$ be a cycle in $H$. Assume $C$ to be odd. Note that $w \notin V(C)$, for if $w \in V(C)$, then $C$ contains a line incident with $w$. But such a line cannot lie on a cycle with $x$ and $y$. Let $z$ be a line of $C$. By definition of $H, z$ must lie on a cycle $C^{\prime}$ containing $x$ and $y$. Let $P_{1}^{\prime}$ and $P_{2}^{\prime}$ be the paths traversed along $C^{\prime}$ by starting at $u$ and $v$ respectively, and stopping upon encountering a point of $C$ for the first time. Let $v_{1}=V(C) \cap V\left(P_{1}^{\prime}\right)$ and $v_{2}=$ $V(C) \cap V\left(P_{2}^{\prime}\right)$. Now $v_{1} \neq v_{2}$ since $C^{\prime}$ contains $z$. Note it may be that $v_{1}=u$ or $v_{2}=v$. The points $v_{1}$ and $v_{2}$ thus induce a division of $C$ into two paths $Q_{1}$ and $Q_{2}$ each of which contains at least one line. But then one of the two cycles $P_{1}^{\prime} \cup P_{2}^{\prime} \cup Q_{1} \cup\{x, y\}$ and $P_{1}^{\prime} \cup P_{2}^{\prime} \cup$ $Q_{2} \cup\{x, y\}$ must be of odd length, contradicting the original assumption, and completing the proof of Assertion 1.

Let $A=\left\{r_{1}, \cdots, r_{m}\right\}$ and $B=\left\{s_{1}, \cdots, s_{n}\right\}$ be the point sets of the bipartite graph $H$ with $u, v \in B$.

Now consider the graph $J=G-H$. The graph $J$ can be partitioned into maximal subgraphs (some perhaps containing no lines), $R_{1}, \cdots R_{m}, S_{1}, \cdots S_{n}$, and $T$ with $r_{i} \in V\left(R_{i}\right), s_{j} \in V\left(S_{j}\right)$, and $w \in V(T)$ 
so that the intersection of any two of these is at most the point $w$. To see this, it need only be shown that any path joining points of $H$ is contained in $H$ unless it contains $w$. This is now proved:

ASSERTION 2. If $u_{0}$ and $u_{1}$ are distinct points of $H$ and if $P$ is a path joining them which does not include $w$, then all lines of $P$ are in $H$.

Let $Q=H+x+y$. Then, clearly, $Q$ is a block and hence $Q \cup P$ is a block. Let $z$ be a line of $P$. Then $z$ and $w$ lie on a common cycle. But the only lines of $Q$ which meet $w$ are $x$ and $y$. Hence $z, x$, and $y$ lie on a common cycle and $z \in H$, proving the assertion.

Thus the graph $G=H \cup J$ has the appearance of Figure 3.

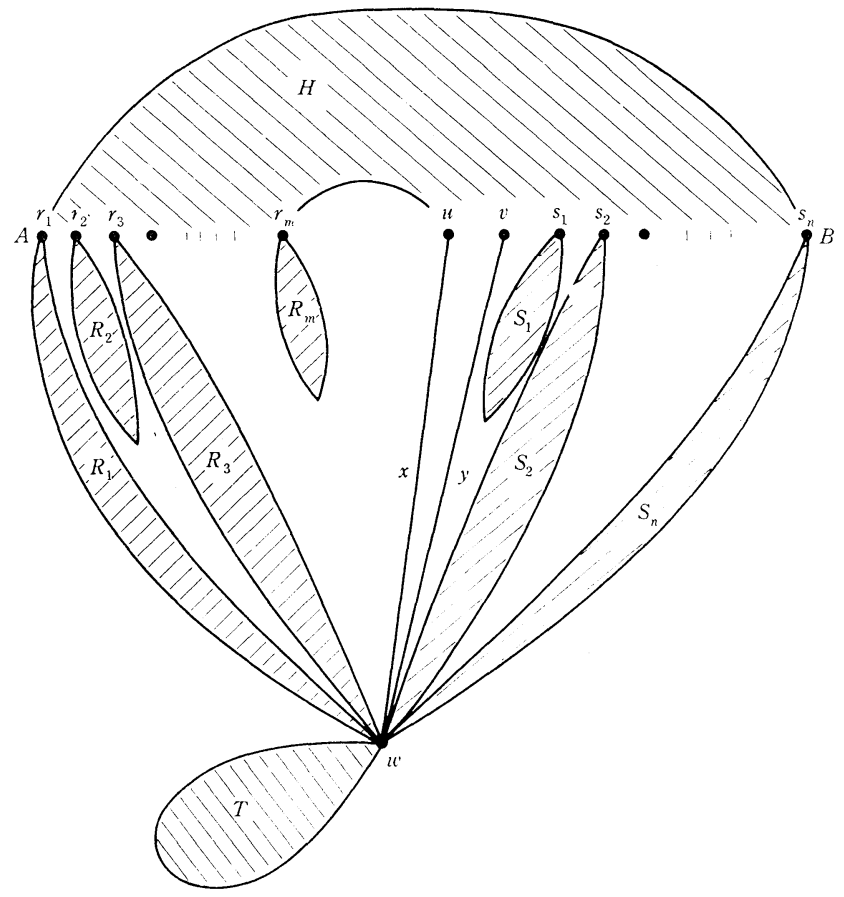

FIGURE 3.

The next objective is to show that m.c.'s for $G-x$ and $G-y$ can be found which meet $V(G)-V(H)$ in exactly the same points. Let $X$ and $Y$ be m.c.'s for $G-x$ and $G-y$ respectively. Hence $u \in Y-X, v \in X-Y$, and $w \notin X \cup Y$. Next note that if there is a point $v^{\prime} \in X \cap Y$, then $X-v^{\prime}$ and $Y-v^{\prime}$ cover $G-x-v^{\prime}$ and $G-y-v^{\prime}$ respectively and hence $x$ and $y$ are both critical in $G-v^{\prime}$.

Now since $w \notin X \cup Y$, both $X \cap V(T)$ and $Y \cap V(T)$ cover $T$. Furthermore, they have the same number of points. Hence it may be assumed that they are identical. 
Next, consider the two sets $X_{i}=X \cap V\left(R_{i}\right)$ and $Y_{i}=Y \cap V\left(R_{i}\right)$ for any $i$. There are exactly three possibilities.

Case 1. Suppose $r_{i} \notin X_{i} \cup Y_{i}$. Then by the same argument as for $T, R_{i}$ is covered by both $X_{i}$ and $Y_{i}$ which must have the same number of points. Hence they may be taken to be equal.

Case 2. Similarly, if $r_{i} \in X_{i} \cap Y_{i}$ it may be assumed that

$$
\left[V\left(R_{i}\right)-\left\{r_{i}\right\}\right] \cap X=\left[V\left(R_{i}\right)-\left\{r_{i}\right\}\right] \cap Y .
$$

Case 3. Finally, suppose $r_{i} \in X_{i}-Y_{i}$. For this case the following statement is required.

\section{Assertion 3. If}

$$
X_{i}=X \cap V\left(R_{i}\right), \quad Y_{i}=Y \cap V\left(R_{i}\right), \quad r_{i}=V(H) \cap V\left(R_{i}\right),
$$

and if $r_{i} \in X_{i}-Y_{i}$, then $\left|Y_{i}\right| \leqq\left|X_{i}\right| \leqq\left|Y_{i}\right|+1$.

Suppose first that $\left|X_{i}\right|<\left|Y_{i}\right|$. Then replace $Y_{i}$ in $Y$ with $X_{i}$. A cover for $G-y$ with fewer elements than $Y$ is thus obtained, contradicting the minimality of $Y$. Hence $\left|Y_{i}\right| \leqq\left|X_{i}\right|$.

Next suppose that $\left|Y_{i}\right|+1<\left|X_{i}\right|$. Then $\left|Y_{i}\right|<\left|X_{i}\right|-1$ and thus $\left|Y_{i} \cup\left\{r_{i}\right\}\right|<\left|X_{i}\right|$. But then $\left[X-X_{i}\right] \cup\left[Y_{i} \cup\left\{r_{i}\right\}\right]$ covers $G-x$ and has fewer elements than $X$, contradicting the minimality of $X$. Hence $\left|X_{i}\right| \leqq\left|Y_{i}\right|+1$ and the assertion is verified.

Now if $\left|Y_{i}\right|=\left|X_{i}\right|, X_{i}$ and $Y_{i}$ may be assumed to be identical as in Case 1. Then it may be assumed that $r_{i} \in X_{i} \cap Y_{i}$. If $\left|X_{i}\right|>$ $\left|Y_{i}\right|$, then $X_{i}$ may be taken to be $Y_{i} \cup\left\{r_{i}\right\}$.

The results of Cases 1, 2, and 3 thus allow the assumption that the points in the symmetric difference $(X-Y) \cup(Y-X)$ are in $H$, i.e., are in $A \cup B$. Now form a new graph $G^{\prime}=G-[(X \cup Y)-(A \cup B)]$. The definition of $G^{\prime}$ allows the presence of isolated points. However, since such points have nothing to do with the point covers of the graph, they will be regarded as deleted. Thus $G^{\prime}$ consists of $H$ together with possibly some lines incident with an $r_{i}$ or an $s_{j}$. Each of these lines is either an endline or is incident with $w$.

The graph $G^{\prime}$ thus has the appearance of Figure 4.

Now clearly $x$ and $y$ lie only on even cycles in $G^{\prime}$. It was pointed out above that if $v^{\prime} \in X \cap Y$, where $X$ and $Y$ are m.c.'s for $G-x$ and $G-y$ respectively, and if $x$ and $y$ are critical in $G$, then $x$ and $y$ remain critical in $G-v^{\prime}$. By repeated application of this property, it follows that $x$ and $y$ are critical in $G^{\prime}$.

Next, build a new graph $G^{\prime \prime}$ from $G^{\prime}$ (cf. Figure 5) by splitting 


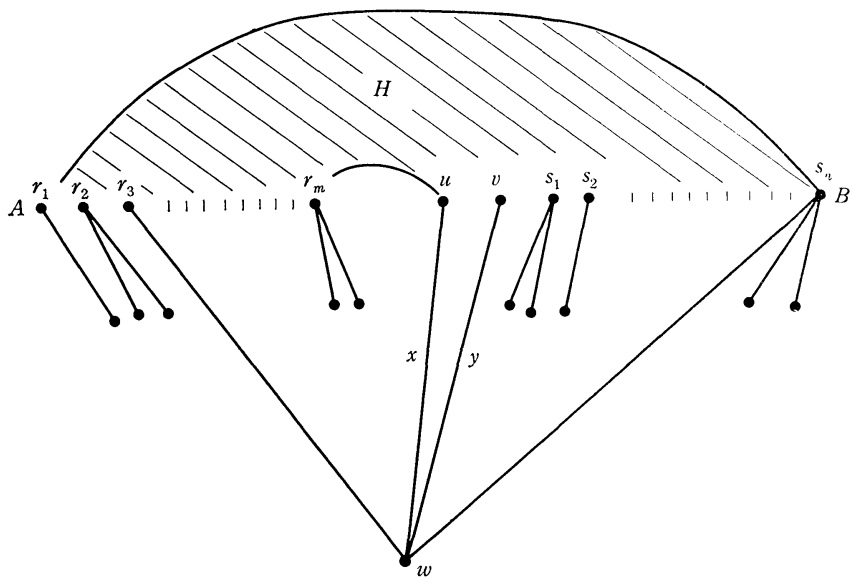

$G^{\prime}$

Figure 4.

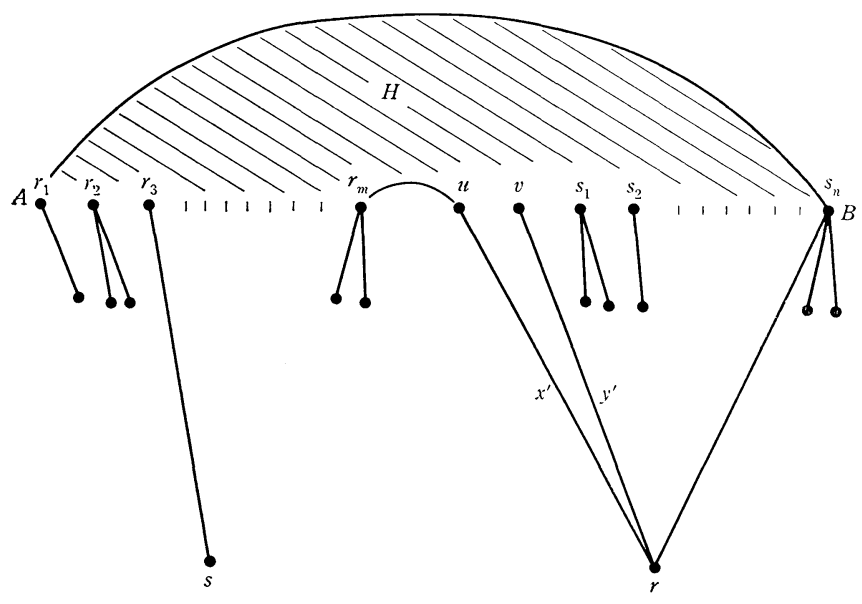

$G^{\prime \prime}$

FIGURE 5.

$w$ into two points $r$ and $s$ such that $r$ is adjacent to those points $s_{j}$ and $s$ to those points $r_{i}$ to which $w$ was adjacent.

In $G^{\prime \prime}$ let $x^{\prime}=u r$ and $y^{\prime}=v r$. It is immediate that $x^{\prime}$ and $y^{\prime}$ are critical in $G^{\prime \prime}$. But the new graph $G^{\prime \prime}$ is bipartite. Hence Theorem 2 is contradicted and the proof of Theorem 3 is complete.

\section{REFERENCES}

1. A. L. Dulmage and N. S. Mendelsohn, Coverings of bipartite graphs, Canad. J. Math. 10 (1958), 517-534.

2. A. L. Dulmage and N. S. Mendelsohn, A structure theory of bipartite graphs of finite exterior dimension, Trans. Roy. Soc. Canada. 53 (1959), 1-13. 
212 LOWELL W. BEINEKE, FRANK HARARY, AND MICHAEL D. PLUMMER

3. P. Erdös and T. Gallai, On the minimum number of vertices representing the edges of a graph, Magyar Tud. Akad. Math. Kutató Int. Közl. 6 (1961), 89-96.

4. O. Ore, Theory of Graphs, Amer. Math. Soc. Colloq. Publ., Vol. 38, Providence, 1962.

5. M. D. Plummer, On a family of line-critical graphs, Monatsh. Math., 71 (1967), $40-48$.

Received December 3, 1965. Work supported in part by the U.S. Air Force Office of Scientific Research under Grant AF-AFOSR-754-65.

UNIVERSITY OF MICHIGAN AND

PURDUE UNIVERSITY 


\section{PACIFIC JOURNAL OF MATHEMATICS}

\section{EDITORS}

\section{H. SAMELSON}

Stanford University

Stanford, California

J. P. JANS

University of Washington

Seattle, Washington 98105

\section{J. DugundJI}

University of Southern California Los Angeles, California 90007

RICHARD ARENS

University of California

Los Angeles, California 90024

\section{ASSOCIATE EDITORS}

E. F. BECKENBACH
B. H. NeUmanN

\section{SUPPORTING INSTITUTIONS}

UNIVERSITY OF BRITISH COLUMBIA CALIFORNIA INSTITUTE OF TECHNOLOGY

UNIVERSITY OF CALIFORNIA

MONTANA STATE UNIVERSITY

UNIVERSITY OF NEVADA

NEW MEXICO STATE UNIVERSITY

OREGON STATE UNIVERSITY

UNIVERSITY OF OREGON

OSAKA UNIVERSITY

UNIVERSITY OF SOUTHERN CALIFORNIA

\author{
STANFORD UNIVERSITY \\ UNIVERSITY OF TOKYO \\ UNIVERSITY OF UTAH \\ WASHINGTON STATE UNIVERSITY \\ UNIVERSITY OF WASHINGTON \\ AMERICAN MATHEMATICAL SOCIETY \\ CHEVRON RESEARCH CORPORATION \\ TRW SYSTEMS \\ NAVAL ORDNANCE TEST STATION
}

Mathematical papers intended for publication in the Pacific Journal of Mathematics should be typewritten (double spaced). The first paragraph or two must be capable of being used separately as a synopsis of the entire paper. It should not contain references to the bibliography. Manuscripts may be sent to any one of the four editors. All other communications to the editors should be addressed to the managing editor, Richard Arens at the University of California, Los Angeles, California 90024.

50 reprints per author of each article are furnished free of charge; additional copies may be obtained at cost in multiples of 50 .

The Pacific Journal of Mathematics is published monthly. Effective with Volume 16 the price per volume ( 3 numbers) is $\$ 8.00$; single issues, $\$ 3.00$. Special price for current issues to individual faculty members of supporting institutions and to individual members of the American Mathematical Society: $\$ 4.00$ per volume; single issues $\$ 1.50$. Back numbers are available.

Subscriptions, orders for back numbers, and changes of address should be sent to Pacific Journal of Mathematics, 103 Highland Boulevard, Berkeley 8, California.

Printed at Kokusai Bunken Insatsusha (International Academic Printing Co., Ltd.), 7-17, Fujimi 2-chome, Chiyoda-ku, Tokyo, Japan.

\section{PUBLISHED BY PACIFIC JOURNAL OF MATHEMATICS, A NON-PROFIT CORPORATION}

The Supporting Institutions listed above contribute to the cost of publication of this Journal, but they are not owners or publishers and have no responsibility for its content or policies. 


\section{Pacific Journal of Mathematics}

\section{Vol. 22, No. $2 \quad$ February, 1967}

Paul Frank Baum, Local isomorphism of compact connected Lie groups ....

Lowell Wayne Beineke, Frank Harary and Michael David Plummer, On the

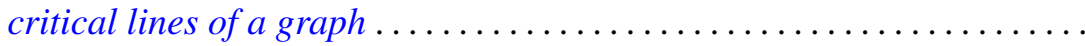

Larry Eugene Bobisud, On the behavior of the solution of the telegraphist's equation for large velocities .......................... 213

Richard Thomas Bumby, Irreducible integers in Galois extensions . . . . . . 221

Chong-Yun Chao, A nonimbedding theorem of nilpotent Lie algebras ..... 231

Peter Crawley, Abelian p-groups determined by their Ulm sequences ...... 235

Bernard Russel Gelbaum, Tensor products of group algebras ........... 241

Newton Seymour Hawley, Weierstrass points of plane domains .......... 251

Paul Daniel Hill, On quasi-isomorphic invariants of primary groups . . . . . 257

Melvyn Klein, Estimates for the transfinite diameter with applications to confomral mapping ................................ 267

Frederick M. Lister, Simplifying intersections of disks in Bing's side approximation theorem ............................. 281

Charles Wisson McArthur, On a theorem of Orlicz and Pettis ........... 297

Harry Wright McLaughlin and Frederic Thomas Metcalf, An inequality for

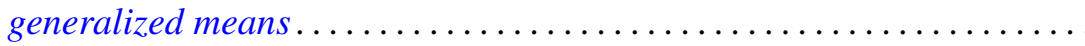

Daniel Russell McMillan, Jr., Some topological properties of piercing

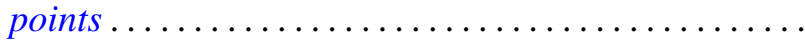

Peter Don Morris and Daniel Eliot Wulbert, Functional representation of topological algebras .

Roger Wolcott Richardson, Jr., On the rigidity of semi-direct products of Lie algebras..................................

Jack Segal and Edward Sandusky Thomas, Jr., Isomorphic

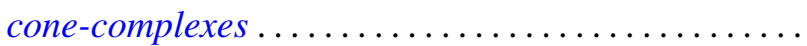

Richard R. Tucker, The $\delta^{2}$-process and related topics.... 